\title{
Functional complementation in Saccharomyces cerevisiae under control of the natural yeast promoter
}

\author{
Rob Lavigne \\ Division of Gene Technology \\ Katholieke Universiteit Leuven \\ Kasteelpark Arenberg 21 \\ Leuven, B-3001, Belgium \\ Tel: 32016329670 \\ Fax: 32016321965 \\ E-mail: rob.lavigne@biw.kuleuven.be \\ Guido Volckaert* \\ Division of Gene Technology \\ Katholieke Universiteit Leuven \\ Kasteelpark Arenberg 21 \\ Leuven, B-3001, Belgium \\ Tel: 32016329667 \\ Fax: 32016321965 \\ E-mail: guido.volckaert@biw.kuleuven.be
}

Financial support: RL holds a postdoctoral fellowship from the FWO (Fonds voor Wetenschappelijk Onderzoek), Vlaanderen.

Keywords: cognate gene plasmid, functional complementation, homolog, Saccharomyces cerevisiae, yeast.

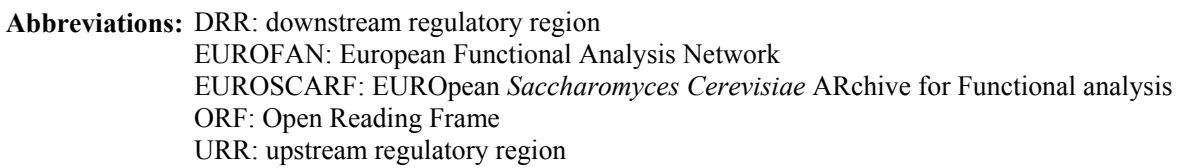

A novel approach for functional complementation of foreign genes in Saccharomyces cerevisiae is presented. This approach is based on the use of the widely available cognate gene plasmids (e.g. pRS416) of the European Functional Analysis Network (EUROFAN). The functional complementation of the human homolog of YOR159c (SME1 gene) shown here is the first demonstration of complementation using the original yeast promoter, theoretically offering a more natural regulation of protein expression.

Functional complementation of yeast mutations by foreign homologues has become a standard practice in functional analysis of Saccharomyces cerevisiae in genes where mutation/disruption provides a phenotypical difference at the cellular level (Agnan et al. 1997). To date, systematic verification of complementation has focused on the availability of vectors containing artificial, inducible promoters (Zhang et al. 2003).

Experiments involving functional complementation should always be treated with a degree of caution, as many aspects of the experimental design may bias the biological outcome of the experiment itself. Well-known problems associated with this type of technique include the relative copy number of the vector being used, episomal replication verses integration, the nature of the antibiotics used to maintain selection, the type, regulation and strength of the promoter chosen (and other transcription and translational regulatory sequences), the distance between promoter and gene and the presence of extraneous sequences.

We present an approach for functional complementation, under control of the original yeast promoter, thus bypassing many of the problems stated above. This approach (Figure 1) is based on the availability of a cognate gene plasmid and closely approximates the natural gene expression in yeast. The $E$. coli $/ S$. cerevisiae shuttle vector pRS416 (URA3 and CEN/ARS cloned in pBlueScript II SK+) was chosen as a standard vector for cloning of replacement cassettes in the European Functional Analysis Network (EUROFAN) (Sikorski and Hieter, 1989). So far, cognate plasmids are available for about $10 \%$ of the 6000 yeast genes, and may be obtained from the EUROSCARF collection index.

\section{MATERIALS AND METHODS}

\section{Construction of pRS416-based cognate gene plasmids}

*Corresponding author 
A cognate gene plasmid was constructed consisting of the pRS416 shuttle-vector in which the yeast open reading frame (ORF) and its regulatory elements were cloned. For YOR159c, the ORF and regulatory elements were amplified by standard PCR (using $P f u$ polymerase and primers YOR159cF and YORcR; Table 1), subsequently cleaved at the Bam HI recognition sites and ligated into the vector. The ligation mixture was directly introduced into a diploid FY1679 YOR159c/DYOR159c. Complementation was verified by sporulation, tetrad dissection, replica plating on selective media, exactly as described previously (Volckaert et al. 2003).

\section{Construction of the pRS416-based complementation plasmid}

The human gene BC002639, homologous to YOR159c, was amplified from an EST clone of the I.M.A.G.E. collection (ID 3609230; obtained from RZPD, the Deutsches Ressourcenzentrum für Genomforschung), containing the full-length cDNA sequence of this gene. PCR amplification of $B C 002639$ was done using primers $\mathrm{BC} 002639 \mathrm{~F}$ and BC002639R (Table 1) and cloned into the $B g / I I$ site in the pRS416-URR/DRR construct (Figure 1). For this construct the URR (upstream regulatory region) and DRR (downstream regulatory region) were amplified using YOR159cF/pOR159ccgplR and YOR159cR/pOR159ccgplF primer couples, respectively (Table 1). After $B g / I I$ digestion, the URR and DRR were spliced, digested again with BamHI and ligated into pRS416 (Figure 1).

The entire construct containing the BC002639 ORF was subsequently transformed to the corresponding heterozygote disruption strain of the yeast ORF. Complementation was verified by sporulation, tetrad dissection, replica plating on selective media and mating type analysis, exactly as described previously (Volckaert et al. 2003).

\section{RESULTS AND DISCUSSION}

The complementation approach was verified on an essential open reading frame YOR159c encoding a protein with a functional human homologue. The YOR159c gene (or SME1 gene) encodes an essential protein (94 aa; $10.4 \mathrm{kDa}$; $\mathrm{pI}=4.38$ similar $45 \%$ identical aa and $74 \%$ similar aa) to the human E-nuclear protein (hSmEp) (Stanford et al. 1988; Bordonné and Tarassov, 1996). Furthermore, it was shown that a cDNA copy of hSmEp under GAL1 promoter control, complements the absence of the SME1 after galactose induction (Bordonné and Tarassov, 1996). SME1 is part of the pre-mRNA spliceosome, where it belongs to the Sm-core, which is essential for the function and biogenesis of the snRNP's.

To confirm the essential nature of YOR159c, we analyzed the chromosomal disruption mutants by PCR (data not shown) and tetrad dissection. The disruption mutant showed growth for only 2 out of 4 spores after sporulation, confirming YOR159c as an essential gene. Episomal complementation of this chromosomal deletion in the cognate gene plasmid, performed as described in the Materials and Methods section, restored growth after sporulation (Figure 2). However, not all spores are viable, which suggests an instability of the plasmid during sporulation. However, since pRS416 is a centromeric vector it should be maintained and suggests this instability is gene specific, since numerous complementations using this vector exist.

A pRS416-based complementation vector for the YOR159c homologue (hSmEp) was constructed as described above. The coding sequence from YOR159c (SME1) is homologous to the human BC002639 gene, encoding $\mathrm{hSmEp}$. Figure 3 shows the human homologue is able to compensate the lack of a wild type yeast gene, when expressed by the original yeast promoter/terminator region, similar to the yeast cognate plasmid.

The successful complementation of YOR159c by the human functional homologue $B C 002639$ acts as a proof of principle that shows functional complementation can be achieved using the original yeast promoter/terminator region. The method presented here only requires the availability of a cognate gene plasmid, constructs widely available within the framework of the EUROFAN project. The method was verified for pRS416, but is independent of this vector and is applicable for any type of cognate plasmid.

A benefit of this method is the possibility to study the human homologues without problems like promoter distance and the presence of extraneous sequences, absent when using the natural promoter/terminator regions. Naturally, special attention should be given to cloning the regulatory regions at the recommended size and is automatically verified when constructing the cognate gene plasmid.

The proof of principle provided here for the human functional homolog of YOR159c is the first demonstration of such a complementation. Although this proof of principle does not show that the levels of expression from native promoters is sufficient to allow complementation in general, this method can be considered a complement to the protocols currently used by the research community.

\section{ACKNOWLEDGMENTS}

The authors thank F. Claes for his contribution as part of his Masters thesis.

\section{REFERENCES}

AGNAN, Jacqueline; KORCH, Christopher and SELITRENNIKOFF, Claude. Cloning heterologous genes: problems and approaches. Fungal Genetics and Biology, June 1997, vol. 21, no. 3, p. 292-301. 
BORDONNÉ, Rémy and TARASSOV, Ivan. The yeast SME1 gene encodes the homologue of the human E core protein. Gene, October 1996, vol. 176, no. 1-2, p. 111-117.

SIKORSKI, Robert S. and HIETER, Philip. A system of shuttle vectors and yeast host strains designed for efficient manipulation of DNA in Saccharomyces cerevisiae. Genetics, May 1989, vol. 122, no. 1, p. 19-27.

STANFORD, David R.; KEHL, Maria; PERRY, Caroline A.; HOLICKY, Eileen L.; HARVEY, Scott E.; ROHLEDER, Anne M.; REHDER, Kai Jr.; LUHRMANN, Reinhard and WIEBEN, Eric D. The complete primary structure of the human snRNP E protein. Nucleic Acids Research, November 1988, vol. 16, no. 22, p. 10593-10605.

VOLCKAERT, Guido; VOET, Marleen; ROBBEN, Johan; VAN DER SCHUEREN, Jan; VANSTREELS, Elisabeth and VANDER STAPPEN, Jacqueline. Disruption of twelve ORFs located on chromosomes IV, VII and XIV of Saccharomyces cerevisiae reveals two essential genes. Yeast, January 2003, vol. 20, no. 1, p. 79-88.

ZHANG, Nianshu; OSBORN, Michael; GITSHAM, Paul; YEN, Kuangyu; MILLER, J. Ross and OLIVER, Stephen G. Using yeast to place human genes in functional categories. Gene, January 2003, vol. 303, p. 121-129. 
Lavigne, R. and Volckaert, G.

\section{APPENDIX \\ FIGURES}

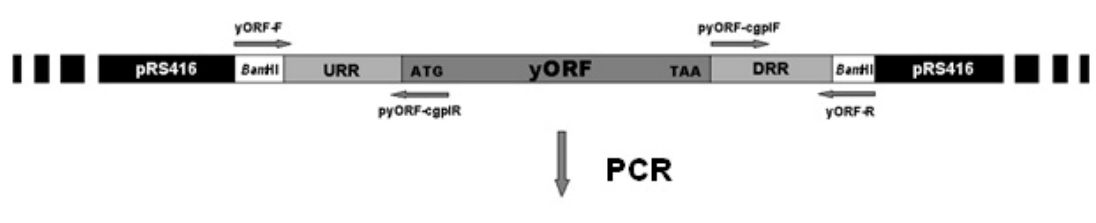

\begin{tabular}{|l|l|l|l|l|l|l|}
\hline Bantil & URR & ATG & Bgnl & Bgil & DRR & Bant1 \\
\hline
\end{tabular}

Ligation and PCR

\begin{tabular}{|l|l|l|l|l|l|}
\hline Bant1 & URR & ATS & Bont & DRR & Bantil \\
\hline
\end{tabular}

Ligation in pRS416

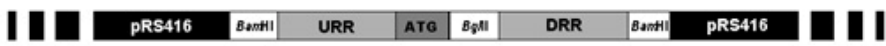

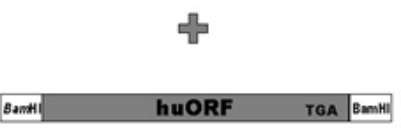

$\mathrm{Bg} / \mathrm{ll}$ digestion and ligation

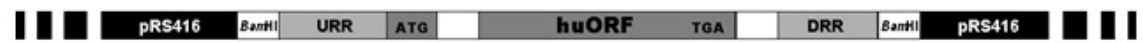

Figure 1. Method for functional complementation under yeast promoter control. Amplification of the Upstream and Downstream Regulatory Regions (URR and DRR) is followed by their ligation. This ligation product is amplified using the yORF-F and yORF-R primers and inserted in pRS416 by BamHI ligation. The human Open reading frame (BC002639) can subsequently be cloned in the $B g / l l$ site between the regulatory regions. Thus, extraneous sequences are limited to 6 bp in the downstream region and 2 codons at the 5 ' of the ORF.
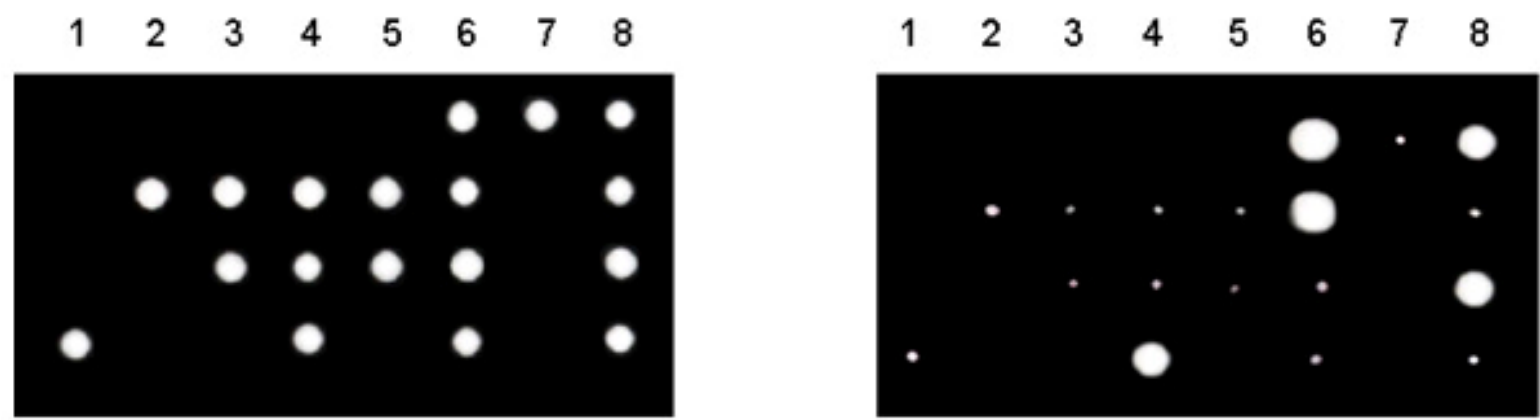

Figure 2. Episomal complementation of YOR159c in pRS416. Tetrad dissection of the FY1679 YOR159c::kanMX/YOR159c strain derived spores, containing the pRS416-YOR159c gene. Complementation is present only in tetrad 6 and 8 where 4 viable spores have grown. (Left on YPD medium; right replica-plating on YPD + geniticin medium). 
a

$$
\begin{array}{llllllllll}
1 & 2 & 3 & 4 & 5 & 6 & 7 & 8 & 9 & 10
\end{array}
$$
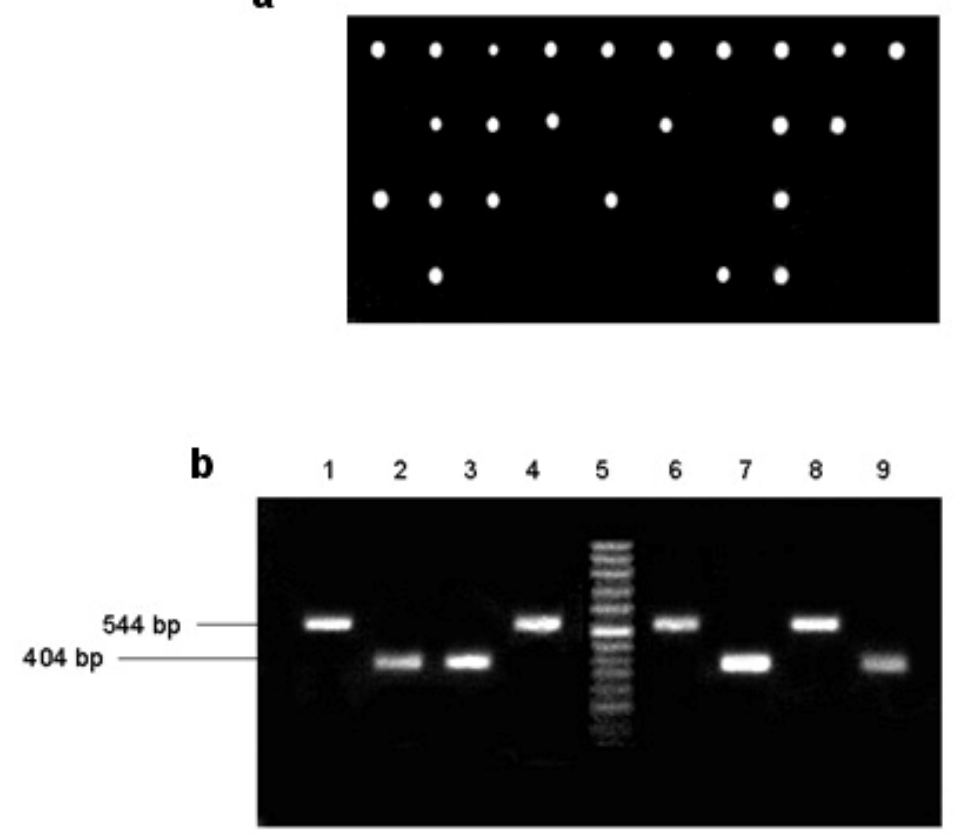

$\begin{array}{llllllllll}1 & 2 & 3 & 4 & 5 & 6 & 7 & 8 & 9 & 10\end{array}$
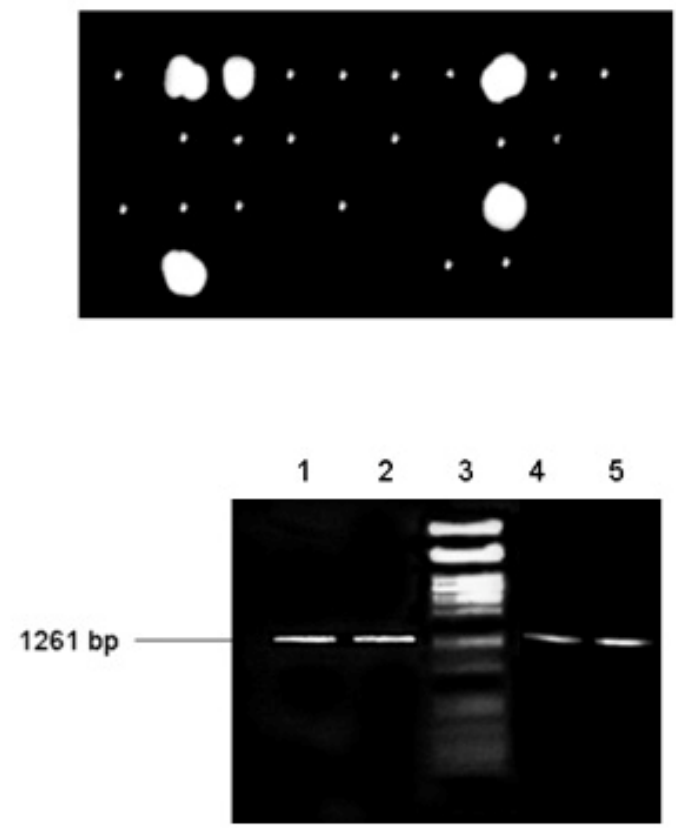

Figure 3. Episomal complementation of the BC002639 gene under yeast promoter control:

(a) Tetrad dissection of the YOR159c::kanMX/YOR159c strain in presence of the human complementation construct on YPD medium (left). Spores in the $2^{\text {nd }}$ and $8^{\text {th }}$ tetrad show a 4:0 segregation between viable and unviable spores. Replica plating of these colonies on YPD geneticin plates show a 2:2 segregation, confirming the functional episomal complementation in the spores containing the KanMX4-deletion cassette (right).

(b) Confirmation of the haplotype of the 4:0 segregated spores of tetrads 2 (lanes 1 to 4 ) and 8 (lanes 6 to 9 ) from a (left). Was done by a standard Mat-locus PCR. For both tetrads, a 2:2 segregation between MAT $\alpha-(544 \mathrm{bp})$ and MATa- (404 bp) spores can be observed. forming $544 \mathrm{bp}$ and $404 \mathrm{bp}$ fragment respectively. Interestingly, all complemented haploid deletion mutants have a MAT $\alpha$ 'mating'-type. Subsequent PCR analysis using T7 and T3 primers confirmed the presence of the plasmid with a correct insert (right). The expected length of the amplicons (1261 bp) for the growing spores of tetrad two (lanes 1-2) and eight (lanes 4-5) are estimated relative to a XPstl ladder (lane 3). 
Lavigne, R. and Volckaert, G.

\section{TABLE}

Table 1. Primers used in this study.

\begin{tabular}{|l|c|}
\hline \multicolumn{1}{|c|}{ Name } & Sequence \\
\hline YOR159cF & 5'-CGGGATCCTCATCTTGAGATGCAG-3' \\
\hline YOR159cR & 5'-CGGGATCCTAACGATGAAACGGAG-3' \\
\hline pOR159ccgplF & 5'-GGTAGATCTAAGAAAAAGGGGAACGA-3' \\
\hline pOR159ccgplR & 5'-GTTAGATCTCATTCCAAGCCTCTTGT-3' \\
\hline BC002639F & 5'-TATGGATCCGCGTACCGTGGCCAGGGTCAG-3' \\
\hline BC002639R & 5'-CCGGGATCCCTAGTTGGAGACACTTTGT-3' \\
\hline T3 & 5'-ATTAACCCTCACTAAAGGGA-3' \\
\hline T7 & 5'- TAATACGACTCACTATAGGG-3' \\
\hline
\end{tabular}

